\title{
Some New Formulae for Genocchi Numbers and Polynomials Involving Bernoulli and Euler Polynomials
}

\author{
Serkan Araci, ${ }^{1}$ Mehmet Acikgoz, ${ }^{2}$ and Erdoğan Şen ${ }^{3,4}$ \\ ${ }^{1}$ Faculty of Economics, Administrative and Social Sciences, Hasan Kalyoncu University, 27410 Gaziantep, Turkey \\ ${ }^{2}$ Department of Mathematics, Faculty of Science and Arts, University of Gaziantep, 27310 Gaziantep, Turkey \\ ${ }^{3}$ Department of Mathematics, Faculty of Science and Letters, Namik Kemal University, 59030 Tekirdağ, Turkey \\ ${ }^{4}$ Department of Mathematics Engineering, Istanbul Technical University, Maslak, 34469 Istanbul, Turkey
}

Correspondence should be addressed to Serkan Araci; mtsrkn@hotmail.com

Received 9 January 2014; Accepted 26 March 2014; Published 24 April 2014

Academic Editor: Ram N. Mohapatra

Copyright (C) 2014 Serkan Araci et al. This is an open access article distributed under the Creative Commons Attribution License, which permits unrestricted use, distribution, and reproduction in any medium, provided the original work is properly cited.

We give some new formulae for product of two Genocchi polynomials including Euler polynomials and Bernoulli polynomials. Moreover, we derive some applications for Genocchi polynomials to study a matrix formulation.

\section{Introduction}

The history of Genocchi numbers can be traced back to Italian mathematician Angelo Genocchi (1817-1889). From Genocchi to the present time, Genocchi numbers have been extensively studied in many different context in such branches of Mathematics as, for instance, elementary number theory, complex analytic number theory, homotopy theory (stable homotopy groups of spheres), differential topology (differential structures on spheres), theory of modular forms (Eisenstein series), $p$-adic analytic number theory ( $p$-adic $L$-functions), and quantum physics (quantum groups). The works of Genocchi numbers and their combinatorial relations have received much attention [1-11]. For showing the value of this type of numbers and polynomials, we list some of their applications.

In the complex plane, the Genocchi numbers, named after Angelo Genocchi, are a sequence of integers that are defined by the exponential generating function:

$$
\frac{2 t}{e^{t}+1}=e^{G t}=\sum_{n=0}^{\infty} G_{n} \frac{t^{n}}{n !}, \quad(|t|<\pi),
$$

with the usual convention about replacing $G^{n}$ by $G_{n}$, is used. When we multiply with $e^{x t}$ in the left-hand side of (1), then we have

$$
\sum_{n=0}^{\infty} G_{n}(x) \frac{t^{n}}{n !}=\frac{2 t}{e^{t}+1} e^{x t}, \quad(|t|<\pi),
$$

where $G_{n}(x)$ are called Genocchi polynomials. It follows from (2) that $G_{1}=1, G_{2}=-1, G_{3}=0, G_{4}=1, G_{5}=0, G_{6}=-3$, $G_{7}=0, G_{8}=17, \ldots$, and $G_{2 n+1}=0$ for $n \in \mathbb{N}$ (for details, see [7-9]).

Differentiating both sides of (1), with respect to $x$, then we have the following:

$$
\frac{d}{d x} G_{n}(x)=n G_{n-1}(x), \quad \operatorname{deg} G_{n+1}(x)=n .
$$

On account of (1) and (3), we can easily derive the following:

$$
\int_{b}^{a} G_{n}(x) d x=\frac{G_{n+1}(a)-G_{n+1}(b)}{n+1} .
$$

By (1), we get

$$
G_{n}(x)=\sum_{k=0}^{n}\left(\begin{array}{l}
n \\
k
\end{array}\right) G_{k} x^{n-k} .
$$



(6):

Thanks to (4) and (5), we acquire the following equation

$$
\int_{0}^{1} G_{n}(x) d x=-2 \frac{G_{n+1}}{n+1} .
$$

It is not difficult to see that

$$
\begin{aligned}
e^{t x} & =\frac{1}{2 t}\left(\frac{2 t}{e^{t}+1} e^{(1+x) t}+\frac{2 t}{e^{t}+1} e^{x t}\right) \\
& =\frac{1}{2 t} \sum_{n=0}\left(G_{n}(x+1)+G_{n}(x)\right) \frac{t^{n}}{n !} .
\end{aligned}
$$

By expression of (7), then we have

$$
2 x^{n}=\frac{G_{n+1}(x+1)+G_{n+1}(x)}{n+1}
$$

(see $[1-25])$.

Let $\mathscr{P}_{n}=\{p(x) \in \mathbb{Q}[x] \mid \operatorname{deg} p(x) \leq n\}$ be the $(n+1)$ dimensional vector space over $\mathbb{Q}$. Probably, $\left\{1, x, x^{2}, \ldots, x^{n}\right\}$ is the most natural basis for $\mathscr{P}_{n}$. From this, we note that $\left\{G_{1}(x), G_{2}(x), \ldots, G_{n+1}(x)\right\}$ is also good basis for space $\mathscr{P}_{n}$.

In [14], Kim et al. introduced the following integrals:

$$
I_{m, n}=\int_{0}^{1} B_{m}(x) x^{n} d x, \quad J_{m, n}=\int_{0}^{1} E_{m}(x) x^{n} d x,
$$

where $B_{m}(x)$ and $E_{n}(x)$ are called Bernoulli polynomials and Euler polynomials, respectively. Also, they are defined by the following generating series:

$$
\begin{aligned}
& e^{B(x) t}=\sum_{n=0}^{\infty} B_{n}(x) \frac{t^{n}}{n !}=\frac{t}{e^{t}-1} e^{x t}, \quad|t|<2 \pi, \\
& e^{E(x) t}=\sum_{n=0}^{\infty} E_{n}(x) \frac{t^{n}}{n !}=\frac{2}{e^{t}+1} e^{x t}, \quad|t|<\pi,
\end{aligned}
$$

with $B^{n}(x):=B_{n}(x)$ and $E^{n}(x):=E_{n}(x)$, symbolically. By (10), then we have

$$
\begin{aligned}
& \frac{t}{e^{t}-1}=\sum_{n=0}^{\infty} B_{n} \frac{t^{n}}{n !} \\
& \frac{2}{e^{t}+e^{-t}}=\sum_{n=0}^{\infty} E_{n} \frac{t^{n}}{n !}
\end{aligned}
$$

Here $B_{n}:=B_{n}(0)$ and $E_{n}:=2^{n} E_{n}(1 / 2)$ are called Bernoulli numbers and Euler numbers, respectively. Additionally, the Bernoulli and Euler numbers and polynomials have the following identities:

$$
B_{n}(x)=\sum_{k=0}^{n}\left(\begin{array}{l}
n \\
k
\end{array}\right) B_{k} x^{n-k}, \quad E_{n}(x)=\sum_{k=0}^{n}\left(\begin{array}{l}
n \\
k
\end{array}\right) E_{k}(0) x^{n-k}
$$

(for details, see $[6,11,13-15,17,19]$ ). By (11), we have the following recurrence relations of Euler and Bernoulli numbers, as follows:

$$
\begin{gathered}
B_{0}=1, \quad B_{n}(1)-B_{n}=\delta_{1, n}, \\
E_{0}=1, \quad(E+1)^{n}+(E-1)^{n}=2 \delta_{0, n},
\end{gathered}
$$

where $\delta_{n, m}$ is the Kronecker's symbol defined by

$$
\delta_{n, m}= \begin{cases}1, & \text { if } n=m \\ 0, & \text { if } n \neq m\end{cases}
$$

In the complex plane, we can write the following:

$$
\sum_{n=0}^{\infty} G_{n} \frac{(i t)^{n}}{n !}=i t \frac{2}{e^{i t}+1}=i t \sum_{n=0}^{\infty} E_{n}(0) \frac{(i t)^{n}}{n !} .
$$

By (15), we have

$$
\sum_{n=0}^{\infty}\left(\frac{G_{n+1}}{n+1}\right) \frac{(i t)^{n}}{n !}=\sum_{n=0}^{\infty} E_{n}(0) \frac{(i t)^{n}}{n !}
$$

by comparing coefficients on the both sides of the above equality, then we have

$$
\frac{G_{n+1}}{n+1}=E_{n}(0)
$$

(see [6]).

Via (17), our results in the present paper can be extended to Euler polynomials at the special value 0 .

Recent works including multiplication formulas for products of Bernoulli and Euler polynomials [17], the product of two Eulerian polynomials [18], sums of products of generalized Bernoulli polynomials [20], explicit formulas for computing Euler polynomials in terms of the second kind Stirling numbers [21], explicit formulas for computing Bernoulli numbers of the second kind and Stirling numbers of the first kind [22], some identities and an explicit formula for Bernoulli and Stirling numbers [23], some identities for the product of two Bernoulli and Euler polynomials [13], some formulae for the product of two Bernoulli and Euler polynomials [14], Bernoulli basis and the product of several Bernoulli polynomials [15], some formulae of products of the Apostol-Bernoulli and Apostol-Euler Polynomials [24], and the modified $q$-Euler numbers of higher order with weight [25] have been extensively investigated.

By the same motivation of the above knowledge, we write this paper. We give some interesting properties which are derived from the basis of Genocchi. From our methods, we obtain not only new but also interesting identities including Bernoulli and Euler polynomials. Also, by using (17), we derive our results in terms of Euler polynomials.

\section{On the Genocchi Numbers and Polynomials}

In this section, we introduce the following integral equation: for $m, n \geq 1$,

$$
T_{m, n}=\int_{0}^{1} G_{m}(x) x^{n} d x .
$$

By (18), becomes

$$
T_{m, n}=-\frac{G_{m+1}}{m+1}-\frac{n}{m+1} \int_{0}^{1} G_{m+1}(x) x^{n-1} d x .
$$

Thus, we have the following recurrence formulas:

$$
T_{m, n}=-\frac{G_{m+1}}{m+1}-\frac{n}{m+1} T_{m+1, n-1},
$$


by continuing with the above recurrence relation; then we derive that

$$
\begin{aligned}
T_{m, n}= & -\frac{G_{m+1}}{m+1}+(-1)^{2} \frac{n}{(m+1)(m+2)} G_{m+2} \\
& +(-1)^{2} \frac{n(n-1)}{(m+1)(m+2)} T_{m+2, n-2} .
\end{aligned}
$$

Now also, we develop the following for sequel of this paper:

$$
T_{m, n}=\frac{1}{n+1} \sum_{j=1}^{n}(-1)^{j} \frac{\left(\begin{array}{c}
n+1 \\
j
\end{array}\right)}{\left(\begin{array}{c}
m+j \\
m
\end{array}\right)} G_{m+j}+2 \frac{(-1)^{n+1} G_{n+m+1}}{(n+m+1)\left(\begin{array}{c}
n+m \\
m
\end{array}\right)} .
$$

Let us now introduce the polynomial

$$
p(x)=\sum_{l=0}^{n} G_{l}(x) x^{n-l}, \quad \text { with } n \in \mathbb{N} .
$$

Taking $k$ th derivative of the above equality, then we have

$$
\begin{aligned}
p^{(k)}(x) & =(n+1) n(n-1) \cdots(n-k+2) \sum_{l=k}^{n} G_{l-k}(x) x^{n-l} \\
& =\frac{(n+1) !}{(n-k+1) !} \sum_{l=k}^{n} G_{l-k}(x) x^{n-l} \quad(k=0,1,2, \ldots, n) .
\end{aligned}
$$

Theorem 1. The following equality holds true:

$$
\begin{aligned}
& \sum_{l=0}^{n} G_{l}(x) x^{n-l} \\
& =\sum_{k=1}^{n-1}\left(\sum_{j=1}^{n-k}(-1)^{j} \frac{\left(\begin{array}{c}
n-k+1 \\
j
\end{array}\right)}{(n-k+1)\left(\begin{array}{c}
k+j \\
k
\end{array}\right)} G_{k+j}\right. \\
& \left.+2 \frac{(-1)^{n-k+1} G_{n+1}}{(n+1)\left(\begin{array}{c}
n \\
k
\end{array}\right)}-2 \frac{G_{k+1}}{k+1}\right) \\
& +\sum_{k=1}^{n}\left(\frac{\left(\begin{array}{c}
n+2 \\
k
\end{array}\right)}{n+2} \sum_{l=k-1}^{n-1}\left(2-G_{l-k+1}-G_{n-k+1}\right)\right) \\
& \quad \times B_{k}(x) .
\end{aligned}
$$

Proof. On account of the properties of the Genocchi basis for the space of polynomials of degree less than or equal to $n$ with coefficients in $\mathbb{Q}$, then $p(x)$ can be written as follows:

$$
p(x)=\sum_{k=0}^{n} a_{k} B_{k}(x)=a_{0}+\sum_{k=1}^{n} a_{k} B_{k}(x) .
$$

Therefore, by (26), we obtain

$$
\begin{aligned}
a_{0} & =\int_{0}^{1} p(x) d x=\sum_{k=1}^{n} \int_{0}^{1} G_{k}(x) x^{n-k} d x \\
& =\sum_{k=1}^{n} T_{k, n-k}=\sum_{k=1}^{n-1} T_{k, n-k}+T_{k, 0}
\end{aligned}
$$

$$
\begin{aligned}
= & \sum_{k=1}^{n-1} \frac{1}{n-k+1} \sum_{j=1}^{n-k}(-1)^{j} \frac{\left(\begin{array}{c}
n-k+1 \\
j
\end{array}\right)}{\left(\begin{array}{c}
k+j \\
k
\end{array}\right)} G_{k+j} \\
& +2 \frac{(-1)^{n-k+1} G_{n+1}}{(n+1)\left(\begin{array}{c}
n \\
k
\end{array}\right)}-2 \frac{G_{k+1}}{k+1} .
\end{aligned}
$$

From expression of (24), we get

$$
\begin{aligned}
a_{k} & =\frac{1}{k !}\left(p^{(k-1)}(1)-p^{(k-1)}(0)\right) \\
& =\frac{(n+1) !}{k !(n-k+2) !}\left(\sum_{l=k-1}^{n} G_{l-k+1}(1)-0^{n-l} G_{n-k+1}\right) \\
& =\frac{\left(\begin{array}{c}
n+2 \\
k
\end{array}\right)}{n+2} \sum_{l=k-1}^{n-1}\left(2-G_{l-k+1}-G_{n-k+1}\right) .
\end{aligned}
$$

Substituting (27) and (28) into (26), we arrive at the desired result.

By using (17) and Theorem 1, we get the following corollary, which has been stated in terms of Euler polynomials.

Corollary 2. For any $n \in \mathbb{N}$, then we have

$$
\begin{aligned}
& \sum_{l=0}^{n} G_{l}(x) x^{n-l} \\
& =\sum_{k=1}^{n-1}\left(\sum_{j=1}^{n-k}(-1)^{j} \frac{(k+j)\left(\begin{array}{c}
n-k+1 \\
j
\end{array}\right)}{(n-k+1)\left(\begin{array}{c}
k+j \\
j
\end{array}\right)} E_{k+j-1}(0)\right. \\
& \left.+2 \frac{(-1)^{n-k+1} E_{n}(0)}{\left(\begin{array}{c}
n \\
k
\end{array}\right)}-2 E_{k}(0)\right) \\
& +\sum_{k=1}^{n}\left(\frac { ( \begin{array} { c } 
{ n + 2 } \\
{ k }
\end{array} ) } { n + 2 } \sum _ { l = k - 1 } ^ { n - 1 } \left(2-(l-k+1) E_{l-k}(0)\right.\right. \\
& \left.\left.-(n-k+1) E_{n-k}(0)\right)\right) B_{k}(x) .
\end{aligned}
$$

Theorem 3. The following nice identity

$$
\begin{aligned}
& \sum_{l=0}^{n} G_{l}(x) x^{n-l} \\
& \quad=\sum_{k=0}^{n}\left((n+1)\left(\begin{array}{l}
n \\
k
\end{array}\right)-\frac{\left(\begin{array}{c}
n+1 \\
k
\end{array}\right)}{2} \sum_{l=k}^{n-1}\left(G_{l-k}-G_{n-k}\right)\right) E_{k}(x)
\end{aligned}
$$

is true.

Proof. Let us now consider the polynomial $p(x)$ in terms of Euler polynomials as follows:

$$
p(x)=\sum_{k=0}^{n} b_{k} E_{k}(x) .
$$


In [14], Kim et al. gave the coefficients $b_{k}$ by utilizing from the definition of Bernoulli polynomials. Now also, we give the coefficients $b_{k}$ by using the definition of Genocchi polynomials, as follows:

$$
\begin{aligned}
b_{k} & =\frac{1}{2 k !}\left(p^{(k)}(1)+p^{(k)}(0)\right) \\
& =\frac{(n+1) !}{2 k !(n-k+1) !} \sum_{l=k}^{n}\left(G_{l-k}(1)+0^{n-l} G_{l-k}\right) \\
& =(n+1)\left(\begin{array}{l}
n \\
k
\end{array}\right)-\frac{\left(\begin{array}{c}
n+1 \\
k
\end{array}\right)}{2} \sum_{l=k}^{n-1}\left(G_{l-k}-G_{n-k}\right) .
\end{aligned}
$$

After the above applications, we complete the proof of the theorem.

By employing (17) and Theorem 3, we have the following corollary, which is the sum of products of two Euler polynomials.

Corollary 4. For each $n \in \mathbb{N}$, one has

$$
\begin{aligned}
& \sum_{l=0}^{n} G_{l}(x) x^{n-l} \\
& =\sum_{k=0}^{n}\left((n+1)\left(\begin{array}{l}
n \\
k
\end{array}\right)\right. \\
& -\frac{\left(\begin{array}{c}
n+1 \\
k
\end{array}\right)}{2} \sum_{l=k}^{n-1}\left((l-k) E_{l-k-1}(0)\right. \\
& \left.\left.-(n-k) E_{n-k-1}(0)\right)\right) E_{k}(x) .
\end{aligned}
$$

We now discover the following theorem, which will be an interesting and worthwhile theorem for studying in analytic numbers theory.

Theorem 5. The following equality holds:

$$
\begin{aligned}
\sum_{l=0}^{n} & \frac{1}{l !(n-l) !} G_{l}(x) x^{n-l} \\
= & \sum_{l=1}^{n} \frac{2^{l-2}}{l !} \sum_{j=l-1}^{n} \frac{\left(2-G_{l-j+1}\right) G_{l}(x)}{(j-l+1) !(n-j) !} \\
& +\frac{2^{l-2}}{l !(n-l+1) !} G_{n-l+1} G_{l}(x) .
\end{aligned}
$$

Proof. It is proved by using the following polynomial $p(x)$ :

$$
p(x)=\sum_{l=0}^{n} \frac{1}{l !(n-l) !} G_{l}(x) x^{n-l}=\sum_{l=0}^{n} a_{l} G_{l}(x) .
$$

It is not difficult to indicate the following:

$$
p^{(k)}(x)=2^{k} \sum_{l=k}^{n} \frac{1}{(l-k) !(n-l) !} G_{l-k}(x) x^{n-l} .
$$

Then, we see that, for $k=1,2, \ldots, n$,

$$
\begin{aligned}
a_{l} & =\frac{1}{2 l !}\left(p^{(l-1)}(1)+p^{(l-1)}(0)\right) \\
& =\frac{2^{l-2}}{l !} \sum_{j=l-1}^{n} \frac{1}{(j-l+1) !(n-j) !}\left(G_{j-l+1}(1)+0^{n-j} G_{j-l+1}\right) \\
& =\frac{2^{l-2}}{l !} \sum_{j=l-1}^{n} \frac{\left(2-G_{l-j+1}\right)}{(j-l+1) !(n-j) !}+\frac{2^{l-2}}{l !(n-l+1) !} G_{n-l+1} .
\end{aligned}
$$

By (35) and (37), we arrive at the desired result.

Theorem 6. The following identity

$$
\begin{aligned}
\sum_{l=0}^{n} & \frac{1}{l !(n-l) !} G_{l}(x) x^{n-l} \\
= & -2 \frac{G_{n+1}}{n+1}+\sum_{l=1}^{n-1} \sum_{j=1}^{n-l} \frac{(-1)^{j}}{l !(n-l+1) !} \frac{\left(\begin{array}{c}
n-l+1 \\
j
\end{array}\right)}{\left(\begin{array}{c}
l+j \\
l
\end{array}\right)} G_{l+j} \\
& +2 \frac{(-1)^{n-l+1} G_{n+1}}{(n+1)\left(\begin{array}{c}
n \\
l
\end{array}\right)} \\
& +\sum_{k=1}^{n}\left(\frac{2^{k-1}}{k !} \sum_{l=k-1}^{n} \frac{\left(2-G_{l-k+1}\right)}{(l-k+1) !(n-l) !}\right. \\
& \left.-\frac{2^{k-1}}{k !(n-k+1) !} G_{n-k+1}\right) B_{k}(x)
\end{aligned}
$$

is true.

Proof. Now also, let us take the polynomial in terms of Bernoulli polynomials as

$$
p(x)=\sum_{k=0}^{n} a_{k} B_{k}(x) .
$$

By using the above identity, we develop as follows:

$$
\begin{aligned}
a_{0}= & \int_{0}^{1} p(x) d x \\
= & \sum_{l=0}^{n} \frac{1}{l !(n-l) !} \int_{0}^{1} G_{l}(x) x^{n-l} d x \\
= & \sum_{l=0}^{n} \frac{1}{l !(n-l) !} T_{l, n-l} \\
= & T_{n, 0}+\sum_{l=1}^{n-1} \frac{1}{l !(n-l) !} T_{l, n-l} \\
= & -2 \frac{G_{n+1}}{n+1}+\sum_{l=1}^{n-1} \sum_{j=1}^{n-l} \frac{(-1)^{j}}{l !(n-l+1) !} \frac{\left(\begin{array}{c}
n-l+1 \\
j
\end{array}\right)}{\left(\begin{array}{c}
l+j \\
l
\end{array}\right)} G_{l+j} \\
& +2 \frac{(-1)^{n-l+1} G_{n+1}}{(n+1)\left(\begin{array}{c}
n \\
l
\end{array}\right)} .
\end{aligned}
$$


By (36), we compute $a_{k}$ coefficients, as follows:

$$
\begin{aligned}
a_{k} & =\frac{1}{k !}\left(p^{(k-1)}(1)-p^{(k-1)}(0)\right) \\
& =\frac{2^{k-1}}{k !} \sum_{l=k-1}^{n} \frac{1}{(l-k+1) !(n-l) !}\left(G_{l-k+1}(1)-0^{n-l} G_{l-k+1}\right) \\
& =\frac{2^{k-1}}{k !} \sum_{l=k-1}^{n} \frac{\left(2-G_{l-k+1}\right)}{(l-k+1) !(n-l) !}-\frac{2^{k-1}}{k !(n-k+1) !} G_{n-k+1}
\end{aligned}
$$

When we substituted (40) and (41) into (39), the proof of theorem will be completed.

By using (17) and Theorem 6, we procure the following corollary.

Corollary 7. For any $n \in \mathbb{N}$, one has

$$
\begin{aligned}
& \sum_{l=0}^{n} \frac{1}{l !(n-l) !} G_{l}(x) x^{n-l} \\
& =-2 E_{n}+\sum_{l=1}^{n-1} \sum_{j=1}^{n-l} \frac{(-1)^{j}}{l !(n-l+1) !} \frac{(l+j)\left(\begin{array}{c}
n-l+1 \\
j
\end{array}\right)}{\left(\begin{array}{c}
l+j \\
l
\end{array}\right)} E_{l+j-1}(0) \\
& +2 \frac{(-1)^{n-l+1} E_{n}(0)}{\left(\begin{array}{c}
n \\
l
\end{array}\right)} \\
& +\sum_{k=1}^{n}\left(\frac{2^{k-1}}{k !} \sum_{l=k-1}^{n} \frac{\left((2 /(l-k+1))-E_{l-k}(0)\right)}{(l-k) !(n-l) !}\right. \\
& \left.-\frac{2^{k-1}}{k !(n-k) !} E_{n-k}(0)\right) B_{k}(x) .
\end{aligned}
$$

In [6], it is well known that

$$
G_{n}(x+y)=\sum_{k=0}^{n}\left(\begin{array}{l}
n \\
k
\end{array}\right) G_{k}(x) y^{n-k} .
$$

For $x=y$ in (43), we have the following:

$$
\frac{1}{n !} G_{n}(2 x)=\sum_{k=0}^{n} \frac{1}{k !(n-k) !} G_{k}(x) x^{n-k}
$$

By comparing the equations of (38) and (44), then we readily derive the following corollary.

\section{Corollary 8. Consider that}

$\frac{1}{n !} G_{n}(2 x)=$ the right-hand-side of equation in Theorem 6 .
Theorem 9. The following equality

$$
\begin{aligned}
\sum_{k=1}^{n-1} & \frac{1}{k(n-k)} G_{k}(x) x^{n-k} \\
= & \sum_{k=0}^{n}\left(\frac{\left(\begin{array}{l}
n \\
k
\end{array}\right)}{2(n-k+1)}\left(H_{n-1}-H_{n-k}\right)\right. \\
& \left.\quad-\frac{\left(\begin{array}{l}
n \\
k
\end{array}\right)}{2 n} \sum_{l=k}^{n-1} \frac{\left(2-G_{l-k+1}\right)}{(n-l)(l-k+1)}\right) G_{k}(x)
\end{aligned}
$$

holds true.

Proof. To prove this theorem, we introduce the following polynomial $p(x)$ :

$$
p(x)=\sum_{k=1}^{n-1} \frac{1}{k(n-k)} G_{k}(x) x^{n-k} .
$$

Then, we derive $k$ th derivative of $p(x)$ that is given by

$$
\begin{aligned}
p^{(k)}(x)= & C_{k}\left(x^{n-k}+G_{n-k}(x)\right)+(n-1)(n-2) \cdots(n-k) \\
& \times \sum_{l=k+1}^{n-1} \frac{G_{l-k}(x) x^{n-l}}{(n-l)(l-k)},
\end{aligned}
$$

where

$$
\begin{array}{r}
C_{k}=\frac{\sum_{j=1}^{k}(n-1) \cdots(n-j+1)(n-j-1) \cdots(n-k)}{n-k} \\
(k=1,2, \ldots, n-1), \quad C_{0}=0 .
\end{array}
$$

We want to note that

$$
\begin{aligned}
p^{(n)}(x) & =\left(p^{(n-1)}(x)\right)^{\prime}=C_{n-1}\left(x+G_{1}(x)\right) \\
& =C_{n-1}=(n-1) ! H_{n-1},
\end{aligned}
$$

where $H_{n-1}$ are called Harmonic numbers, which are defined by

$$
H_{n-1}=\sum_{j=1}^{n-1} \frac{1}{j}
$$

With the properties of Genocchi basis for the space of polynomials of degree less than or equal to $n$ with coefficients in $\mathbb{Q}, p(x)$ is introduced by

$$
p(x)=\sum_{k=0}^{n} a_{k} G_{k}(x) .
$$


By expression of (52), we obtain that

$$
\begin{aligned}
a_{k}= & \frac{1}{2 k !}\left(p^{(k-1)}(1)+p^{(k-1)}(0)\right) \\
= & \frac{C_{k-1}}{2 k !}\left(1+2 \delta_{1, n-k+1}\right) \\
& +\frac{(n-1) !}{2 k !(n-k) !} \sum_{l=k}^{n-1} \frac{\left(G_{l-k+1}(1)+0^{n-l} G_{l-k+1}\right)}{(n-l)(l-k+1)} \\
= & \frac{C_{k-1}}{2 k !}-\frac{\left(\begin{array}{l}
n \\
k
\end{array}\right)}{2 n} \sum_{l=k}^{n-1} \frac{\left(2-G_{l-k+1}\right)}{(n-l)(l-k+1)} .
\end{aligned}
$$

As a result,

$$
a_{n}=\frac{1}{2 n !}\left(p^{(n)}(1)+p^{(n)}(0)\right)=\frac{C_{n-1}}{n !}=\frac{H_{n-1}}{n} .
$$

In [14], it is well known that

$$
\frac{C_{k-1}}{k !}=\frac{\left(\begin{array}{c}
n \\
k
\end{array}\right)}{(n-k+1)}\left(H_{n-1}-H_{n-k}\right) .
$$

By (48), (52), and (55), we arrive at the desired result.

From (17) and Theorem 9, we acquire the following.

Corollary 10. The following identity holds:

$$
\begin{aligned}
& \sum_{k=1}^{n-1} \frac{1}{k(n-k)} G_{k}(x) x^{n-k} \\
& =\sum_{k=1}^{n}\left(\frac{\left(\begin{array}{l}
n \\
k
\end{array}\right)}{2(n-k+1)}\left(H_{n-1}-H_{n-k}\right)\right. \\
& \left.\quad-\frac{\left(\begin{array}{l}
n \\
k
\end{array}\right)}{2 n} \sum_{l=k}^{n-1} \frac{\left((2 /(l-k+1))-E_{l-k}(0)\right)}{(n-l)}\right) \\
& \quad \times k E_{k-1}(x) .
\end{aligned}
$$

\section{Further Remarks}

Let $\mathscr{P}_{n}=\left\{\sum_{j=0} a_{j} x^{j} \mid a_{j} \in \mathbb{Q}\right\}$ be the space of polynomials of a degree less than or equal to $n$. In this final section, we will give the matrix formulation of Genocchi polynomials. Let us now consider the polynomial $p(x) \in \mathscr{P}_{n}$ as a linear combination of Genocchi basis polynomials with

$$
p(x)=C_{1} G_{1}(x)+C_{2} G_{2}(x)+\cdots+C_{n+1} G_{n+1}(x) .
$$

We can write the above as a product of two variables:

$$
p(x)=\left(\begin{array}{llll}
G_{1}(x) & G_{2}(x) & \cdots & G_{n+1}(x)
\end{array}\right)\left(\begin{array}{c}
C_{1} \\
C_{2} \\
\vdots \\
C_{n+1}
\end{array}\right) .
$$

From expression of (58), we consider the following equation:

$$
\begin{aligned}
p(x)= & \left(\begin{array}{lllll}
1 & x & x^{2} & \cdots & x^{n}
\end{array}\right)\left(\begin{array}{cccc}
g_{1,1} & g_{1,2} & \cdots & g_{1, n+1} \\
0 & g_{2,2} & \cdots & g_{2, n+1} \\
0 & 0 & \cdots & g_{3, n+1} \\
\vdots & \vdots & \ddots & \vdots \\
0 & 0 & 0 & g_{n+1, n+1}
\end{array}\right) \\
& \times\left(\begin{array}{c}
C_{1} \\
C_{2} \\
C_{3} \\
\vdots \\
C_{n+1}
\end{array}\right),
\end{aligned}
$$

where $g_{i, j}$ are the coefficients of the power basis that are used to determine the respective Genocchi polynomials. We now list a few Genocchi polynomials as follows:

$$
\begin{gathered}
G_{1}(x)=1, \quad G_{2}(x)=2 x-1, \\
G_{3}(x)=3 x^{2}-3 x, \quad G_{4}(x)=4 x^{3}-6 x^{2}-1, \ldots
\end{gathered}
$$

In the quadratic case $(n=2)$, the matrix representation is

$$
p(x)=\left(\begin{array}{lll}
1 & x & x^{2}
\end{array}\right)\left(\begin{array}{ccc}
1 & -1 & 0 \\
0 & 2 & -3 \\
0 & 0 & 3
\end{array}\right)\left(\begin{array}{l}
C_{1} \\
C_{2} \\
C_{3}
\end{array}\right) .
$$

In the cubic case $(n=3)$, the matrix representation is

$$
p(x)=\left(\begin{array}{llll}
1 & x & x^{2} & x^{3}
\end{array}\right)\left(\begin{array}{cccc}
1 & -1 & 0 & -1 \\
0 & 2 & -3 & 0 \\
0 & 0 & 3 & -6 \\
0 & 0 & 0 & 4
\end{array}\right) .
$$

Remark 11. Throughout this paper, many considerations for Genocchi polynomials seem to be useful to study in a matrix formulation.

\section{Conflict of Interests}

The authors declare that there is no conflict of interests regarding the publication of this paper.

\section{Acknowledgment}

The authors would like to thank an anonymous referee for his/her valuable comments and suggestions which have improved the presentation of the paper.

\section{References}

[1] S. Araci, "Novel identities for $q$-Genocchi numbers and polynomials," Journal of Function Spaces and Applications, vol. 2012, Article ID 214961, 13 pages, 2012.

[2] S. Araci, "Novel identities involving Genocchi numbers and polynomials arising from applications from umbral calculus," Applied Mathematics and Computation, vol. 233, pp. 599-607, 2014. 
[3] S. Araci, E. Sen, and M. Acikgoz, "Theorems on Genocchi polynomials of higher order arising from Genocchi basis," Taiwanese Jouurnal of Mathematics, vol. 18, no. 2, pp. 473-482, 2014.

[4] S. Araci, M. acikgoz, A. Bagdasaryan, and E. Şen, “The Legendre polynomials associated with Bernoulli, Euler, Hermite and Bernstein polynomials," Turkish Journal of Analysis and Number Theory, vol. 1, no. 1, pp. 1-3, 2013.

[5] S. Araci, M. Acikgoz, and E. Şen, "On the extended Kim's padic $q$-deformed fermionic integrals in the $p$-adic integer ring," Journal of Number Theory, vol. 133, no. 10, pp. 3348-3361, 2013.

[6] T. Kim, "Some identities for the Bernoulli, the Euler and the Genocchi numbers and polynomials," Advanced Studies in Contemporary Mathematics, vol. 20, no. 1, pp. 23-28, 2010.

[7] A. Genocchi, "Intorno all' espressione generale de' Numeri Bernulliani," Annali Di SciEnzE MatEmatichE E FisichE, vol. 3, pp. 395-405, 1852.

[8] A. F. Horadam, "Genocchi polynomials," in Proceedings of the 4th International Conference on Fibonacci Numbers and Their Applications, pp. 145-166, Kluwer Academic, 1991.

[9] A. F. Horadam, "Negative order Genocchi polynomials," The Fibonacci Quarterly, vol. 30, no. 1, pp. 21-34, 1992.

[10] H. M. Srivastava, B. Kurt, and Y. Simsek, "Some families of Genocchi type polynomials and their interpolation functions," Integral Transforms and Special Functions, vol. 23, no. 12, pp. 919-938, 2012.

[11] H. M. Srivastava, "Some generalizations and basic (or q-) extensions of the Bernoulli, Euler and Genocchi polynomials," Applied Mathematics \& Information Sciences, vol. 5, no. 3, pp. 390-444, 2011.

[12] T. Kim, "Symmetry of power sum polynomials and multivariate fermionic p-adic invariant integral on $\mathbb{Z}_{p}$," Russian Journal of Mathematical Physics, vol. 16, no. 1, pp. 93-96, 2009.

[13] D. S. Kim, T. Kim, S. H. Lee, and Y. H. Kim, "Some identities for the product of two Bernoulli and Euler polynomials," Advances in Difference Equations, vol. 2012, article 95, 2012.

[14] D. S. Kim, D. V. Dolgy, T. Kim, and S.-H. Rim, "Some formulae for the product of two Bernoulli and Euler polynomials," Abstract and Applied Analysis, vol. 2012, Article ID 784307, 15 pages, 2012.

[15] D. S. Kim and T. Kim, "Bernoulli basis and the product of several Bernoulli polynomials," International Journal of Mathematics and Mathematical Sciences, vol. 2012, Article ID 463659, 12 pages, 2012.

[16] V. Gupta, T. Kim, J. Choi, and Y. H. Kim, "Generating functions for $g$-Bernstein, $g$-Meyer-König-Zeller and $g$-Beta basis," Automation, Computers, Applied Mathematics, vol. 19, no. 1, pp. 7-11, 2010.

[17] L. Carlitz, "Multiplication formulas for products of Bernoulli and Euler polynomials," Pacific Journal of Mathematics, vol. 9, pp. 661-666, 1959.

[18] L. Carlitz, "The product of two Eulerian polynomials," Mathematics Magazine, vol. 36, no. 1, pp. 37-41, 1963.

[19] H. M. Srivastava and J. Choi, Series Associated with the Zeta and Related Functions, Kluwer Academic Publishers, Dordrecht, The Netherlands, 2001.

[20] K.-W. Chen, "Sums of products of generalized Bernoulli polynomials," Pacific Journal of Mathematics, vol. 208, no. 1, pp. 39-52, 2003.

[21] F. Qi, "Explicit formulas for computing Euler polynomials in terms of the second kind Stirling numbers," http://arxiv.org/abs/1310.5921 .
[22] F. Qi, "Explicit formulas for computing Bernoulli numbers of the second kind and Stirling numbers of the first kind," Filomat, vol. 28, pp. 1-9, 2014.

[23] B.-N. Guo and F. Qi, "Some identities and an explicit formula for Bernoulli and Stirling numbers," Journal of Computational and Applied Mathematics, vol. 255, pp. 568-579, 2014.

[24] Y. He and C. Wang, "Some formulae of products of the ApostolBernoulli and Apostol-Euler polynomials," Discrete Dynamics in Nature and Society, vol. 2012, Article ID 927953, 11 pages, 2012.

[25] S.-H. Rim and J. Jeong, "On the modified $q$-Euler numbers of higher order with weight," Advanced Studies in Contemporary Mathematics, vol. 22, no. 1, pp. 93-98, 2012. 


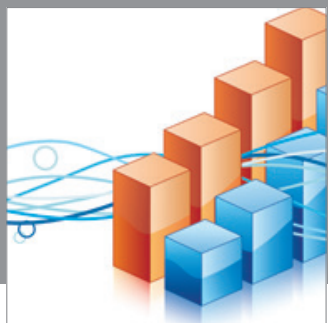

Advances in

Operations Research

mansans

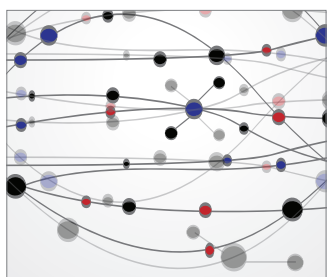

The Scientific World Journal
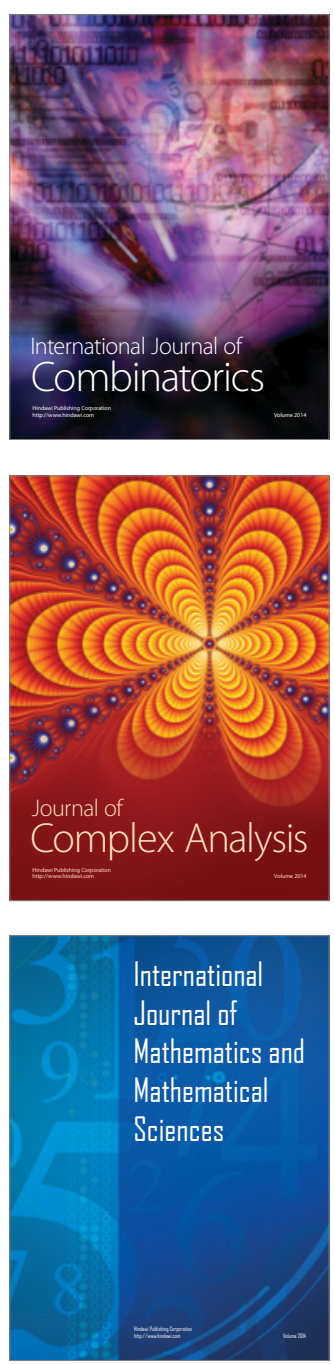
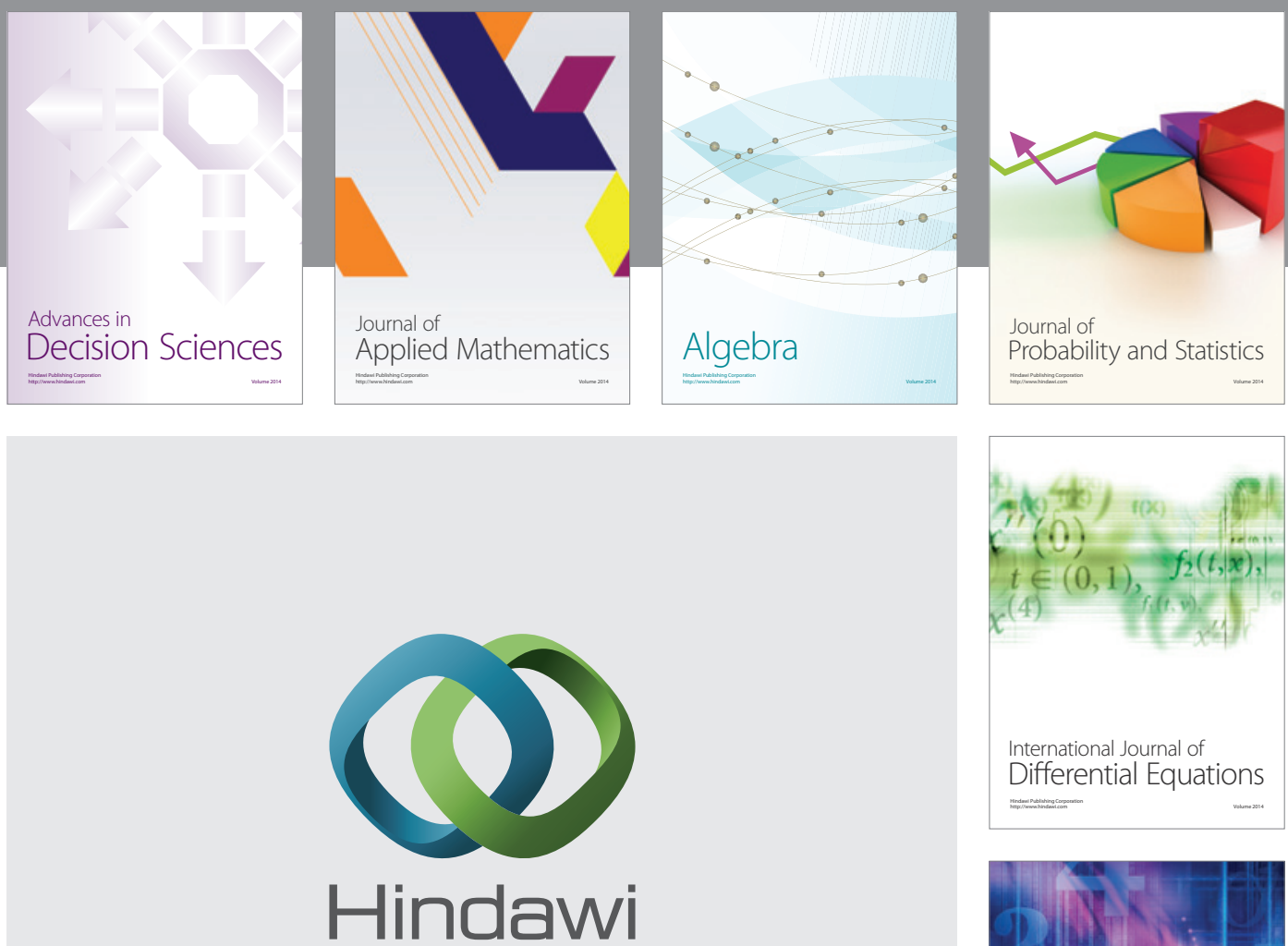

Submit your manuscripts at http://www.hindawi.com
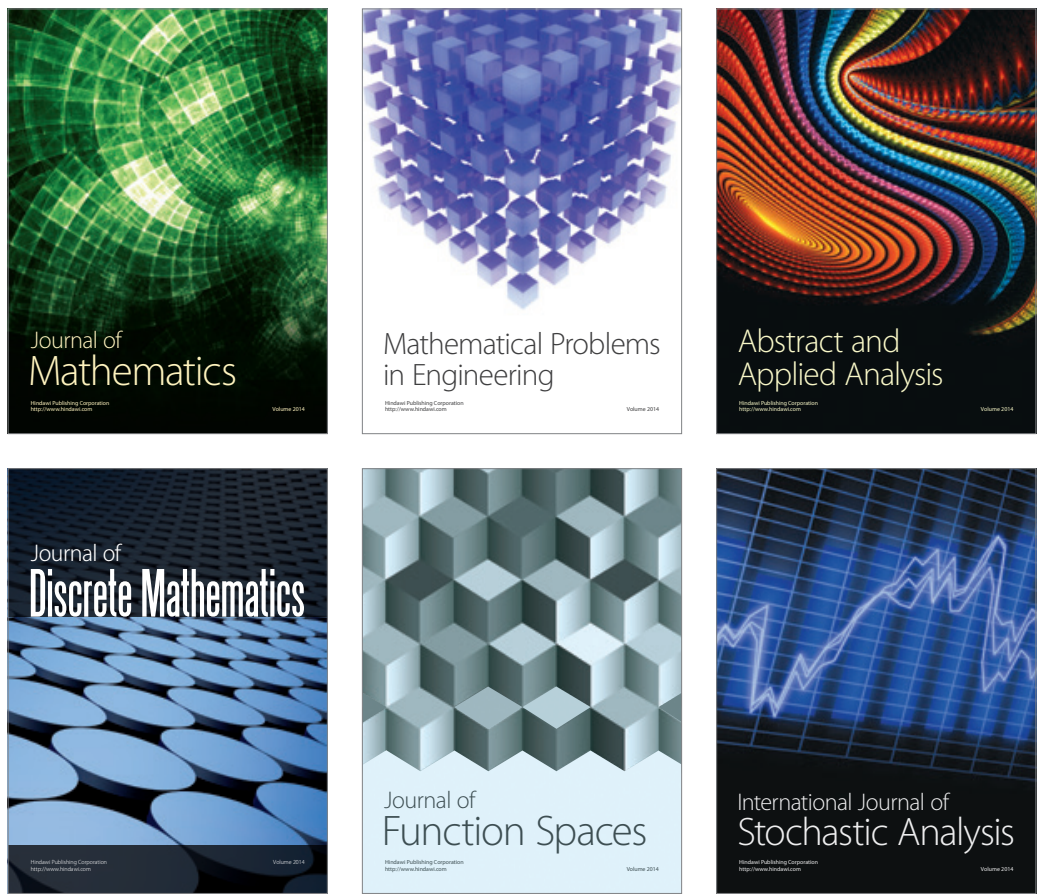

Journal of

Function Spaces

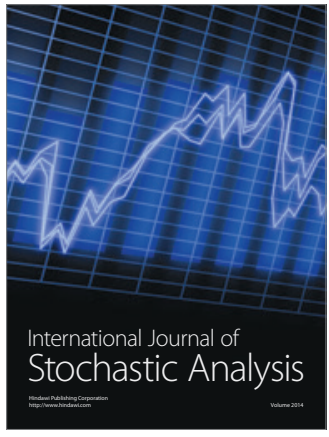

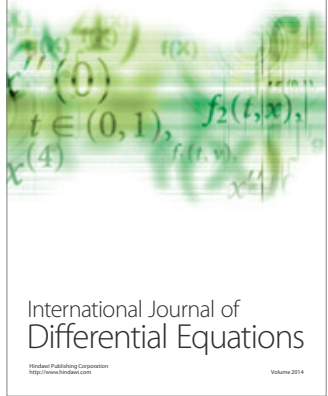
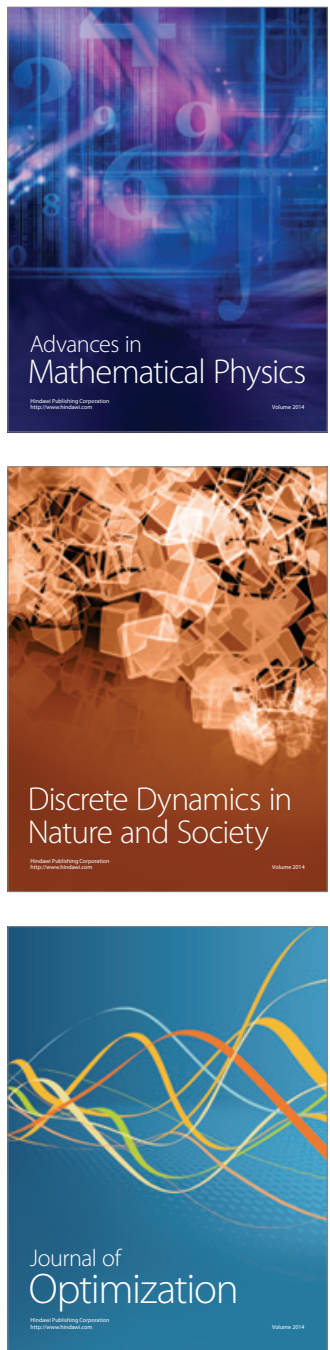